\title{
Determinación de proteínas: un recurso diagnóstico para evaluar sueros de bovinos con paratuberculosis
}

\section{Protein determination: a diagnostic resource to evaluate sera from cattle with paratuberculosis}

\author{
Miceli Graciela Sara ${ }^{1, *}$, Alvarado Pinedo María Fiorella ${ }^{2}$ ， Moyano Roberto \\ Damián 3 , Travería Gabriel Eduardo ${ }^{1,2}$
}

1. Cátedra de Inmunología Veterinaria Aplicada. Facultad de Ciencias Veterinarias, Universidad Nacional de La Plata (FCV-UNLP). 2. Centro de Diagnóstico e Investigaciones Veterinarias (CEDIVE). 3. Consejo Nacional de Investigaciones Científicas y Técnicas (CONICET). Laboratorio de Paratuberculosis. Instituto de Biotecnología, Instituto Nacional de Tecnología Agropecuaria, INTA Castelar, provincia de Buenos Aires, Argentina.

* Correo electrónico de la autora de contacto: gmiceli@fcv.unlp.edu.ar

\begin{abstract}
Resumen
Este trabajo analiza las proteínas séricas de bovinos procedentes de un rodeo con paratuberculosis endémica, enfermedad infecciosa asociada a diarrea, pérdida de estado corporal, caquexia y muerte. Se comparan los resultados obtenidos a partir de un grupo de animales positivos a tres pruebas diagnósticas de paratuberculosis con otro grupo negativo. Las pruebas utilizadas para formar los grupos fueron el cultivo mico-bacteriológico de materia fecal, la inoculación intradérmica con tuberculina aviar y un ELISA indirecto para detección de anticuerpos en suero. La concentración de las proteínas séricas y sus fracciones se analizaron mediante colorimetría y electroforesis. Los intervalos de credibilidad bayesianos fueron estadísticamente diferentes entre los grupos positivo y negativo a las pruebas diagnósticas, con menores concentraciones de albúmina y mayor concentración de gammaglobulina. La relación albúmina/globulina también presentó diferencias significativas, con disminución de la relación en el grupo positivo. Las fracciones proteicas alfa y beta no presentaron diferencias. La disminución de albúmina en el grupo de animales positivos justifica la reducción de la eficiencia productiva y enfatiza la conveniencia de segregarlos animales positivos, no solo por las pérdidas económicas asociada a la enfermedad, sino también como una medida de control para evitar la difusión de la misma.
\end{abstract}

\section{Palabras clave}

Paratuberculosis bovina, albúmina, globulina, electroforesis

\begin{abstract}
Bovine serum proteins were analyzed in sera from bovines from a herd with endemic paratuberculosis, an infectious disease associated with diarrhea, loss of body condition, cachexia and death. A group of negative animals was compared with a group of positive animals to 3 diagnostic tests for paratuberculosis (mycobateriological culture of feces, intradermal inoculation of avian tuberculin and antibody detection by indirect ELISA). Serum protein concentrations and their different fractions were analyzed by the colorimetric method and electrophoresis. Bayesian credible intervals were statistically different, with a lower albumin concentration and higher gamma globulin concentration in the positive group compared to the negative group. The albumin/globulin ratio was also statistically different with decreased ratio in the positive group. No statistical differences were observed in alpha and beta protein fractions. The decreased albumin in the positive group partly explains the poor productive performance and emphasizes the convenience to test and cull positive animals not only to avoid economic losses, but also as a control measure to reduce dissemination of the disease.
\end{abstract}

\section{Key words}

Bovine paratuberculosis, albumin, globulin, electrophoresis

Fecha de recepción: 11/03/2019

Fecha de revisión: 13/05/2019

Fecha de aprobación: 26/06/2019
ANALECTA Vet 2019; Julio-Diciembre; 39(2):2-7

Impresa ISSN 03655 14-8 Electrónica ISSN 1514-2590

doi.org/10.24215/15142590eo37 


\section{Introducción}

La paratuberculosis (PTB) bovina es una enfermedad infectocontagiosa producida por la micobacteria Mycobacterium avium subsp. paratuberculosis (Map) (Over et al., 2011). Los animales afectados presentan ileocolitis granulomatosa de curso crónico. Los signos clínicos más prominentes en los bovinos consisten en diarrea progresiva, pérdida de peso y producción láctea, emaciación y muerte (Whitlock \& Buergelt, 1996). La mayoría de los animales se infecta en los primeros meses de vida, siendo el período de incubación de la enfermedad de 2 a 8 años (Koets et al., 2015). Los terneros se infectan vía fecal-oral, al tomar contacto con los animales enfermos, los cuales pueden eliminar la micobacteria por la materia fecal, la leche o el calostro, incluso antes de presentar signos clínicos (Mortier et al., 2015; Sweeney, 1996). La forma de controlar la enfermedad se basa en el diagnóstico y la segregación de los animales positivos y en la implementación de medidas de manejo tendientes a evitar la infección de los animales jóvenes (Gautam et al., 2018). No se conocen tratamientos efectivos y las vacunas pueden interferir en el diagnóstico de la tuberculosis (Park \& Yoo, 2016), siendo esta última la razón por la que no están permitidas, por el momento, en la Argentina.

Los cambios en las proteínas séricas pueden indicar procesos patológicos no específicos que afecten hígado, riñón y tracto gastrointestinal. Las proteínas séricas se han utilizado como biomarcadores en diversas enfermedades, tales como bronconeumonías, metritis, mastitis y pericarditis traumáticas, entre otras. Entre las fracciones proteicas analizadas se encuentran la albúmina, la gammaglobulina y las proteínas de fase aguda (Tothova et al., 2016). En la PTB bovina, a consecuencia de la enteropatía generada, se produce un síndrome de malabsorción y mala digestión (Sweeney, 2011) con alteraciones cuantitativas en las proteínas séricas (Donat et al., 2014; You et al., 2012).

El objetivo del presente trabajo fue demostrar si existen diferencias en la concentración de proteínas y sus fracciones proteicas analizadas mediante electroforesis en acetato de celulosa, entre animales positivos y negativos a las pruebas diagnósticas de PTB bovina.

\section{Materiales y métodos}

Se analizaron 16 muestras de suero seleccionadas de 127 animales pertenecientes a un establecimiento ubicado en el partido de Magdalena, provincia de Buenos Aires, con PTB bovina endémica. En el mismo, se está implementando un plan de control, consistente en el sangrado periódico para la realización de un ELISA indirecto, la prueba tuberculínica anocaudal con PPD aviar y el aislamiento de Map. El lote original estaba compuesto por cinco toros y 122 hembras bovinas Aberdeen Angus adultas, preñadas, con cría al pie, alimentados en pasturas naturales e implantadas. El plan sanitario está basado en vacunaciones anuales para fiebre aftosa, carbunclo, canbunclo sintomático y brucelosis; se complementa con desparasitaciones, tratamientos para la queratoconjuntivitis y suplementación con cobre, de acuerdo con el criterio del profesional veterinario sanitarista. La reposición del rodeo proviene del propio establecimiento y de reproductores adquiridos en remates de cabañas.

A partir del suero de los 127 animales se realizó un ELISA indirecto, de acuerdo con lo publicado previamente por Costanzo et al. (2012). Brevemente, se utilizó como antígeno un lisado completo de Mycobacterium avium subsp. avium cepa D4ER cultivado en caldo glicerinado durante un período de 2 meses. El soma bacteriano filtrado se inactivó mediante irradiación gamma a una dosis de 20 unidades Gray. La lisis del soma bacteriano inactivado se realizó en un disruptor celular (Constant Systeminc ${ }^{\circledR}$, Inglaterra) a una presión de $40 \mathrm{Kpsi}\left(1000 \mathrm{lb} / \mathrm{in}^{2}\right)$. Posteriormente, el lisado se centrifugó a $10.000 \mathrm{~g}$ durante 20 minutos y el sobrenadante se utilizó como antígeno lisado completo. Cada uno de los 96 pocillos de las microplacas de ELISA (Microlon ${ }^{\circledR}$, Greiner bio-one, Kremsmünster, Austria) se sensibilizó con $100 \mu \mathrm{l}$ del antígeno diluido $1 / 480$ en solución tamponada de carbonatos a pH 9, durante 12 horas a $4^{\circ} \mathrm{C}$. Las placas sensibilizadas se lavaron 3 veces con una solución tamponada de fosfatos con 0,05 \% de Tween20 a pH 7,2 (solución tamponada ELISA) y se incubaron con $100 \mu \mathrm{l}$ de los sueros problema y control diluidos $1 / 160$ en solución tamponada ELISA durante 30 minutos a temperatura ambiente. Después de 3 lavados con esta solución, se incubaron con $100 \mu \mathrm{l}$ de conjugado anti IgG bovina-peroxidasa (Sigma-Aldrich ${ }^{\circledR}$, Darmstadt, Alemania) a una dilución de 1/5000 en solución tamponada ELISA durante 30 minutos a temperatura ambiente. Por último se realizaron 3 lavados y se agregaron $100 \mu \mathrm{l}$ de la solución de revelado compuesta por $1 \mathrm{mg} / \mathrm{ml}$ de ABTS $(-2,2$ azino-di-[3-ethyl-benzothiazoline-6-sulphonic acid], Sigma-Aldrich, St Louis, MO, USA) en solución tamponada de citrato a $\mathrm{pH} 5 \mathrm{y}$ con el agregado de peróxido de hidrógeno al $3 \%$. Transcurridos 30 minutos, se realizó la lectura en un espectrofotómetro (Labsystem, Multiskan plus ${ }^{\circledR}$, Helsinki, Finlandia) con un filtro de 405 $\mathrm{nm}$. Se consideraron positivos los sueros con un valor igual o superior a $70 \%$ con respecto al control positivo.

Para la prueba tuberculínica se inoculó en forma intradérmica, en el pliegue anocaudal interno, o,1 $\mathrm{ml}$ de PPD aviar en una concentración de $0,5 \mathrm{mg} / \mathrm{ml}$ (Ceva Salud Animal ${ }^{\circledR}$, Buenos Aires, Argentina), utilizando una jeringa semiautomática (McLintock Bar Knigth $®$, Glasgow, Reino Unido); transcurridas las 72 horas, se realizó la lectura midiendo la diferencia en $\mathrm{mm}$ del pliegue cutáneo 
inoculado con respecto al opuesto, utilizando un calibre de metal, la metodología previamente descripta de aplicación de la tuberculina y de la lectura sigue las normativas de la Resolución de tuberculosis 128/2012 de SENASA (SENASA, 2012). En paratuberculosis, para definir a los animales reactantes a la tuberculina aviar al momento de la lectura, se consideraron a quienes presentaron alguna diferencia en el pliegue cutáneo según lo sugerido por Alvarado Pinedo (2015).

Para el aislamiento de Map en materia fecal se recolectaron muestras de los 127 animales. Se colocaron 2 gramos de cada muestra en $30 \mathrm{ml}$ de agua destilada estéril en tubos de polipropileno de $50 \mathrm{ml}$ con tapa a rosca, y se agitó durante 30 segundos para disgregar el material. Transcurridos 30 minutos de reposo en posición vertical para permitir el sedimento del material fibroso, se transfirieron $5 \mathrm{ml}$ del sobrenadante a otro tubo de polipropileno conteniendo $25 \mathrm{ml}$ de una solución al $0,9 \%$ de cloruro de hexadecilpiridinio (SigmaAldrich ${ }^{\circledR}$ Darmstadt, Alemania). Las muestras se incubaron durante 12 horas a $37^{\circ} \mathrm{C}$. Posteriormente, se centrifugaron a $900 \mathrm{~g}$ durante $30 \mathrm{minu}-$ tos, se descartó el sobrenadante y al sedimento se agregó $1 \mathrm{ml}$ de una solución compuesta por caldo infusión cerebro corazón diluido al $50 \%$, con el agregado de vancomicina $100 \mathrm{mg} / \mathrm{ml}$, ácido nalidíxico $100 \mathrm{mg} / \mathrm{ml}$ y nistatina $200 \mathrm{mg} / \mathrm{ml}$. Las muestras se incubaron durante 24 horas a $37^{\circ} \mathrm{C}$. Por último, se transfirieron $100 \mu \mathrm{l}$ a un tubo de vidrio con tapa a rosca conteniendo $4 \mathrm{ml}$ de un medio de cultivo líquido con micobactina $\mathrm{J}$ (Allied Monitor Inc ${ }^{\circledR}$, Missouri, Estados Unidos) a razón de $1 \mathrm{mg} / \mathrm{l}$ del medio de cultivo (Stabel, 1997; Whittington et al., 2013). Las muestras se incubaron durante 2 meses, con tinciones periódicas de los medios de cultivo líquido, con el método de Ziehl Neelsen para la observación de bacilos ácido alcohol resistentes, compatibles con Map.

De acuerdo con los resultados obtenidos en las pruebas diagnósticas de PTB bovina de los 127 bovinos estudiados, se seleccionaron 16 animales divididos en un grupo positivo y otro negativo, para realizar la determinación en suero de proteínas mediante colorimetría y electroforesis (Tabla 1).

Tabla 1. Criterios de selección de los 16 animales analizados para la determinación de proteínas séricas.

\begin{tabular}{cccc}
\hline $\begin{array}{c}\text { Resultados de las } \\
\text { pruebas diagnósticas } \\
\text { de paratuberculosis }\end{array}$ & Prueba de ELISA & $\begin{array}{c}\text { Prueba intradérmica } \\
\text { con PPD aviar }\end{array}$ & Cultivo de Map \\
\hline 8 positivos & $8 / 8$ positivos & $6 / 8$ reactantes* & $8 / 8$ positivos \\
8 negativos & $8 / 8$ negativos & $8 / 8$ no reactantes & $8 / 8$ negativos \\
\hline
\end{tabular}

*Los reactantes a la PPD aviar presentaron diferencias de tamaño de 2 a $6 \mathrm{~mm}$, en el engrosamiento del pliegue cutáneo (Alvarado Pinedo, 2015).
La determinación de proteínas totales y albúmina se realizó mediante colorimetría utilizando el kit comercial Proti2 ${ }^{\circledR} \quad$ (Wiener Laboratorios S.A.I.C., Rosario, Argentina), de acuerdo con las instrucciones del fabricante.

La electroforesis se realizó sobre cintas de cellogel $(2,5 \times 17 \mathrm{~cm}, 250 \mu \mathrm{m})$, sembrándose las muestras con aplicador semimicro $\left(\mathrm{Chemar}^{\circledR}\right.$, Capital Federal, Argentina). Las cintas se colocaron en una cubeta de electroforesis (Labnet International ${ }^{\circledR}$, Taiwan) a $35 \mathrm{~mA}$ y $200 \mathrm{~V}$ en buffer borato-acetato, pH 8,6 (Biopur ${ }^{\circledR}$ S.R.L., Rosario, Argentina) (Heer \& Margni, 1971). Finalizada la corrida, las cintas se colorearon con Amido Black $10 \mathrm{~B}$, se decoloraron con una solución de agua destilada, ácido acético y metanol 79:79:8,5 ml, se cortaron las distintas fracciones y se realizó su elusión. Luego, se calcularon los valores de las fracciones, relacionándolas con el valor de las proteínas totales.

$\mathrm{El}$ análisis estadístico entre los grupos se realizó usando el software R versión 3.2.3 (R Core Team, 2015) con el paquete BEST (Kruschke, 2013), una versión bayesiana de la prueba de $T$, analizando la distribución posterior de las medias, tomando como diferencias significativas al intervalo de credibilidad bayesiano (ICB) que no contiene al cero.

\section{Resultados}

Las proteínas totales en los sueros del grupo negativo al aislamiento de Map presentaron una media de $7,7 \mathrm{~g} \%$ con un ICB de 6,8 a $8,5 \mathrm{~g} \%$. El grupo positivo al aislamiento de Map presentó valores de $8 \mathrm{~g} \%$ con ICB de 7,2 a 8,7 g\%, con una diferencia entre los sueros de $0,3 \mathrm{~g} \%$ y un ICB de $-0,8$ a 1,5 g\%; dado que el intervalo contiene al cero, las diferencias se consideran como no significativas.

La albúmina en el grupo negativo presentó valores de $5 \mathrm{~g} \%$. En el grupo positivo los valores disminuyeron a $2,8 \mathrm{~g} \%$ con ICB de 2,6 a $2,9 \mathrm{~g} \%$, con una diferencia significativa de $-2,2 \mathrm{~g} \% \mathrm{y}$ un ICB de $-2,6$ a $-2,8$ g\%.

La gammaglobulina en el grupo de sueros procedentes de animales con pruebas diagnósticas negativas, presentaron una media de 1,3 g\%. En el grupo de animales positivos la media se ubicó en $3,7 \mathrm{~g} \%$, con un aumento significativo de $2,4 \mathrm{~g} \% \mathrm{y}$ un ICB de 1,5 a 3,3 g\%. Estos resultados se presentan en la Tabla 2.

La relación albúmina/globulina en el grupo negativo a las pruebas diagnósticas presentó una media de 2. En los sueros positivos la relación disminuyó a 0,5 con una diferencia significativa de $-1,5$ con un ICB de $-2,1$ a $-0,9$ g\%, como se muestra en la tabla 2 y en la figura 1, en las que se observa que el cero se encuentra fuera del ICB.

Tanto en las fracciones proteicas beta como en las alfa, no se encontraron diferencias significativas entre los grupos (Tabla 2). 
Tabla 2. Resultados de las diferencias en las fracciones proteicas entre los grupos de animales positivos y negativos a las pruebas diagnósticas de paratuberculosis bovina analizados con estadística bayesiana.

\begin{tabular}{lcccc}
\hline \multicolumn{1}{c}{ Proteínas séricas } & $\begin{array}{c}\text { Medias del } \\
\text { grupo negativo a } \\
\text { PTB (ICB) }\end{array}$ & $\begin{array}{c}\text { Medias del } \\
\text { grupo positivo } \\
\text { a PTB (ICB) }\end{array}$ & $\begin{array}{c}\text { Diferencia } \\
\text { entre las } \\
\text { medias (ICB) }\end{array}$ & $\begin{array}{c}\text { Valores de } \\
\text { referencia ** }\end{array}$ \\
\hline Proteínas totales en g\% & $7,7(6,8 ; 8,5)$ & $8(7,2 ; 8,7)$ & $0,3(-0,8 ; 1,5)$ & $6-8$ \\
Albúmina en g\%* & $5(4,6 ; 5,4)$ & $2,8(2,6 ; 2,9)$ & $-2,2(-2,6 ;-1,8)^{*}$ & $3-4$ \\
$\alpha$-globulinas en g\% & $0,5(0,3 ; 0,8)$ & $0,4(0,2 ; 0,6)$ & $-0,1(-0,4 ; 0,2)$ & 0,79 \\
$\beta$ - globulinas en g\% & $0,7(0,1 ; 1,3)$ & $0,9(0,1 ; 1,7)$ & $0,2(-0,7 ; 1,3)$ & 0,96 \\
$\gamma$ - globulinas en g\%* & $1,3(1,1 ; 1,5)$ & $3,7(2,8 ; 4,6)$ & $2,4(1,5 ; 3,3)^{*}$ & 1,97 \\
Relación A/G* & $2(1,4 ; 2,7)$ & $0,5(0,4 ; 0,6)$ & $-1,5(-2,1 ;-0,9)^{*}$ & $0,70-0,83$ \\
\hline
\end{tabular}

* Diferencia significativa debido a que el intervalo de credibilidad bayesiano (ICB) no contiene al o, tomando al ICB como límite superior e inferior con un $95 \%$ de credibilidad. ** De acuerdo con Kaneko et al. (2008) y Miceli et al. (2014).

\section{Discusión y conclusiones}

Debido al carácter crónico de la enfermedad, el diagnóstico de los animales con PTB requiere del empleo de una serie de pruebas diagnósticas repetidas a intervalos regulares (Meyer et al., 2019). La prueba más utilizada para detectar la respuesta inmune humoral representada por los anticuerpos es el ELISA. La mayoría de los animales positivos a esta prueba son asintomáticos (Collins et al., 2005). Sin embargo, los resultados obtenidos demuestran que los sueros analizados mediante electroforesis presentan diferencias en la composición de las proteínas séricas, con una franca disminución de 2 g\% en la fracción de la albúmina en los animales que tienen anticuerpos contra Map con respecto a los que no los tienen. La disminución en la albúmina sérica representa probables consecuencias negativas en el desempeño productivo de los animales afectados, como lo demuestra el edema submandibular que suele acompañar a los animales enfermos, a causa de una disminución en la presión oncótica generada por la hipoalbuminemia (Nielsen \& Toft, 2008; Whittington et al., 2017). En la fracción de las inmunoglobulinas la diferencia es de 2,4 g\% a favor de los animales con anticuerpos contra Map a causa de la respuesta inmune de tipo humoral. Esta diferencia en los niveles de la albúmina y las inmunoglobulinas altera la relación albúmina/ globulina, generando una disminución significativa de $-1,5$. Esta proporción resulta de particular interés, ya que su variación es indicativa de disproteinemia; los valores normales se ubican en un rango de 0,70- 0,83 (Kaneko et al., 1997; Nagy et al., 2015). En nuestro estudio, la relación albúmina/globulina en los animales negativos a PTB bovina resultó mayor a los valores normales de referencia. Esto pudo deberse a diversos factores tales como estado general, nutrición, edad, sexo o diferencias metodológicas de las pruebas utilizadas. La PTB bovina es causa de severas lesiones en el íleon y en el colon, causando un síndrome de malabsorción, con disminución de la producción láctea y la ganancia de peso (Sweeney,
2011). En las enfermedades que afectan al tracto gastrointestinal, como la PTB bovina, la relación albúmina/globulina se encuentra alterada por la deficiencia en la albúmina y la respuesta inmune humoral crónica con producción de inmunoglobulinas (Kaneko et al., 2008).

\section{Diferencia de medias}

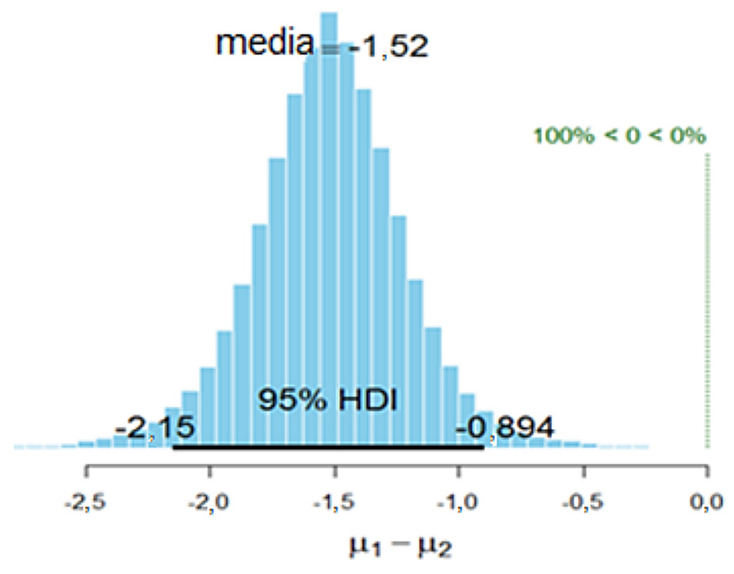

Figura 1. Representación gráfica de la diferencia de medias en la relación albúmina/globulina entre el grupo de animales positivos y negativos a las pruebas diagnósticas de paratuberculosis. La barra horizontal negra representa el $95 \%$ HDI (Highest Density Intervals) con sus intervalos de credibilidad bayesiano (ICB) $95 \%(-2,15,-0,894)$, el ICB no contiene al cero; $\mu 1$ : media del grupo 1 (positivos a las pruebas diagnósticas), $\mu 2$ : media del grupo 2 (negativos a las pruebas diagnósticas). El $100 \%$ de los valores se encuentran a la izquierda del cero, demostrando una disminución significativa en la relación albúmina/globulina.

Cuando se analizaron las proteínas totales, no se encontraron diferencias debido a que la disminución de la albúmina fue compensada con el aumento de la gammaglobulina; las fracciones alfa y beta globulinas no presentaron diferencias con los métodos empleados. Los resultados obteni- 
dos en este estudio aportan información original sobre los niveles cuantitativos de las proteínas séricas en los bovinos con PTB, permitiendo correlacionarlos con los distintos estadios de esta enfermedad y con los resultados de las pruebas diagnósticas utilizadas.

En este estudio, los animales expuestos a la infección con Map, detectados mediante ELISA y, en 6 de ellos también mediante la reacción a la tuberculina, además fueron eliminadores del agente infeccioso en las heces, tal como lo demuestran los aislamientos a partir de la materia fecal. La presencia de bovinos adultos infectados eliminadores de micobacterias, ponen en riesgo a los animales jóvenes, la categoría más susceptible a la infección. Los programas de control de la enfermedad se basan en el diagnóstico y la segregación de los animales positivos (Roussel, 2011). Si los animales positivos no se excluyen de los establecimientos rurales, se prolonga el tiempo de los programas de control, con las consecuentes pérdidas productivas, los costos derivados de las pruebas diagnósticas y la infección de nuevos individuos (Shrestha et al., 2018). La detección de animales enfermos en los diferentes estadios requiere de la realización e interpretación de varias pruebas diagnósticas. Las pruebas que se utilizan en forma rutinaria en Argentina son el ELISA y la tuberculina aviar por las ventajas operativas que presentan, entre las que se puede mencionar el costo y, para el caso del ELISA, la fácil obtención de las muestras (Costanzo et al., 2012). Los resultados obtenidos demuestran que los animales detectados mediante estas técnicas, en su mayoría se encuentran eliminando el agente causal y presentan alteraciones en sus proteínas séricas, lo que probablemente afecte en forma negativa la producción, justificando la segregación de los animales positivos.

\section{Agradecimientos}

Los autores agradecen la colaboración del propietario del establecimiento rural utilizado en este estudio. Este estudio fue parcialmente financiado por el Proyecto de Investigación y Desarrollo acreditado por la UNLP V220.

\section{Conflicto de intereses}

Todos los autores declaran que no existe conflicto de intereses, incluyendo las relaciones financieras, personales o de otro tipo con otras personas u organizaciones que pudieran influir de manera inapropiada en el trabajo.

\section{Bibliografía}

Alvarado Pinedo MF. 2015. Posibilidades diagnósticas de la PPD aviar en la paratuberculosis bovina en animales jóvenes. Tesis doctoral Universidad Nacional de La Plata. Disponible en: http://sedici.unlp.edu.ar/handle/10915/48228

Collins MT, Wells SJ, Petrini KR, Collins JE, Schultz RD, Whitlock RH. 2005. Evaluation of five antibody detection tests for diagnosis of bovine paratuberculosis. Clinical and Diagnostic Laboratory Immunology. 12(6):685-92.

doi: 10.1128/CDLI.12.6.685-692.2005

Costanzo G, Pinedo FA, MonML, Viale M, Gil A, Illia MC, Gioffré A, Arese A, Travería G, Romano MI. 2012. Accuracy assessment and screening of a dairy herd with paratuberculosis by three different ELISAs. Veterinary Microbiology. 156(1-2):183-8. doi: 10.1016/j.vetmic.2011.10.029

Donat K, Erhardt G, Soschinka A, Brandt HR. 2014. Decreased serum protein associated with Mycobacterium avium subspecies paratuberculosis shedding in German Holstein cows. Veterinary Record. 174(16):408.

doi: 10.1136/vr.101957

Gautam M, Ridler A, Wilson PR, Heuer C. 2018. Control of clinical paratuberculosis in New Zealand pastoral livestock. New Zealand Veterinary Journal. 66(1):1-8.

doi: 10.1080/00480169.2017.1379914

Heer E, Margni R. 1971. Electro e inmunoelectroforesis. Manual de laboratorio e interpretaciones fundamentales. Buenos Aires, Gumersindo F. Fernández.

Kaneko JJ, Harvey JW, Bruss ML.1997. Serum proteins and the dysproteinemias. En:Kaneko JJ (Ed):Clinical biochemistry of domestic animals.5 ta Ed. London, Academic Press, pp. 117-38.

Kaneko JJ,Harvey JW, BrussML. 2008. Clinical biochemistry of domestic animals. 6ta Ed. London. Academic Press.

Koets AP, Eda S, Sreevatsan S. 2015. The within host dynamics of Mycobacterium avium ssp. paratuberculosis infection in cattle: where time and place matter. Veterinary Research. 46(1):61. doi: 10.1186/s13567-015-0185-0

Kruschke JK. 2013. Bayesian estimation supersedes the $\mathrm{t}$ test. Journal of Experimental Psychology. 142(2):573-603.

doi: $10.1037 / \mathrm{a} 0029146$

Meyer A, McAloon CG, Tratalos JA, More SJ, Citer LR, Graham DA, Sergeant ESG. 2019. Modeling of alternative testing strategies to demonstrate freedom from Mycobacterium avium ssp. paratuberculosis infection in test-negative dairy herds in the Republic of Ireland. Journal of Dairy Science. 102(3):2427-42. doi: 10.3168/jds.2018-14883 
Miceli G, Bonzo E, Fontana L. 2014. Electroforesis en acetato de celulosa: comparación de resultados. XX Reunión Científica y Técnica de la Asociación Argentina de Veterinarios de Laboratorio de Diagnóstico. Tucumán, Argentina, p. 57.

Mortier RA, Barkema HW, De Buck J. 2015. Susceptibility to and diagnosis of Mycobacterium avium subspecies paratuberculosis infection in dairy calves: A review. Preventive Veterinary Medicine. 121(3-4):189-98.

doi: 10.1016/j.prevetmed.2015.08.011

Nielsen SS, Toft N. 2008. Ante mortem diagnosis of paratuberculosis: a review of accuracies of ELISA, interferon-gamma assay and faecal culture techniques. Veterinary Microbiology. 129(3-4): 217-35. doi: 10.1016/j.vetmic.2007.12.011

Nagy O,Tothová C, Nagyova V, Kovac G. 2015. Comparison of serumprotein electrophoretic pattern in cow and small rumiants. Acta Veterinaria Brno. 84:187-195.

doi: 10.2754/avb201584020187

Over K, Crandall PG, O'Bryan CA, Ricke SC. 2011. Current perspectives on Mycobacterium avium subsp. paratuberculosis, Johne's disease, and Crohn's disease: a review. Critical Reviews in Microbiology. 37(2):141-56.

doi: 10.3109/1040841X.2010.532480

Park HT, Yoo HS. 2016. Development of vaccines to Mycobacterium avium subsp. paratuberculosis infection. Clinical and Experimental Vaccine Research. 5(2):108-16.

doi: 10.7774/cevr.2016.5.2.108

$\mathrm{R}$ Core Team. 2015. R: A language and environment for statistical computing. $\mathrm{R}$ Foundation for Statistical Computing, Vienna, Austria.URL https://www.R-project.org/

Roussel AJ. 2011.Control of paratuberculosis in beef cattle. Veterinary Clinics of North America Food Animal Practice. 27(3):593-8.

doi: 10.1016/j.cvfa.2011.07.005

SENASA 2012. Disponible en: http:// www.senasa.gob.ar/sites/default/files/ARBOL SENASA/INFORMACION/NORMATIVA/ RESOL_Y_ANEXOS/reso_128_articulos_1107.pdf. [Consultado 16/02/2019]

Shrestha S, Vosough Ahmadi B, Barratt AS, Thomson SG, Stott AW. 2018. Financial vulnerability of dairy farms challenged by Johne's disease to changes in farm payment support.
Frontiers in Veterinary Science. 5:316.

doi: 10.3389/fvets.2018.00316

Stabel JR. 1997. An improved method for cultivation of Mycobacterium paratuberculosis from bovine fecal samples and comparison to three other methods. Journal of Veterinary Diagnostic Investigation. 9(4):375-80.

doi: 10.1177/104063879700900406

Sweeney RW. 2011. Pathogenesis of paratuberculosis. Veterinary Clinics of North America Food Animal Practice. 27(3):537-46.

doi: 10.1016/j.cvfa.2011.07.001

Sweeney RW. 1996. Transmission of paratuberculosis. Veterinary Clinics of North America Food Animal Practice. 12 (2):305-12. doi: 10.1016/So749-0720(15)30408-4

Tothova C, Nagy O, Kovac G.2016. Serum proteins and their diagnostic utility in veterinary medicine: a review. Veterinarni Medicina. 61(9):475-96. doi: 10.17221/19/2016-VETMED

Whitlock RH, Buergelt C. 1996. Preclinical and clinical manifestations of paratuberculosis (including pathology). Veterinary Clinics of North America Food Animal Practice. 12(2):345-56. doi: 10.1016/So749-0720(15)30410-2

Whittington RJ, Whittington AM, Waldron A, Begg DJ, de Silva K, Purdie AC, Plain KM. 2013. Development and validation of a liquid medium (M7H9C) for routine culture of Mycobacterium avium subsp. paratuberculosis to replace modified Bactec 12B medium. Journal of Clinical Microbiology. 51(12):3993-4000.

doi: 10.1128/JCM.01373-13

Whittington RJ, Begg DJ, de Silva K, Purdie AC, Dhand NK, Plain KM. 2017. Case definition terminology for paratuberculosis (Johne's disease). BMC Veterinary Research. 13(1):328. doi: 10.1186/s12917-017-1254-6

You Q, Verschoor CP, Pant SD, Macri J, Kirby GM, Karrow NA. 2012. Proteomic analysis of plasma from Holstein cows testing positive for Mycobacterium avium subsp. paratuberculosis (MAP). Veterinary Immunology and Immunopathology. 148(3-4):243-51. doi: 10.1016/j.vetimm.2012.05.002 